\title{
Exploring the selectivity of WO3 with iridium catalyst in an ethanol/naphthalene mixture using multivariate statistics
}

Manuel Bastuck, Donatella Puglisi, J. Huotari, T. Sauerwald, J. Lappalainen, Anita Lloyd Spetz, Mike Andersson and A. Schuetze

\section{Journal Article}

\section{Tweet}

N.B.: When citing this work, cite the original article.

Original Publication:

Manuel Bastuck, Donatella Puglisi, J. Huotari, T. Sauerwald, J. Lappalainen, Anita Lloyd Spetz, Mike Andersson and A. Schuetze, Exploring the selectivity of WO3 with iridium catalyst in an ethanol/naphthalene mixture using multivariate statistics, Thin Solid Films, 2016. 618, pp.263-270.

http://dx.doi.org/10.1016/j.tsf.2016.08.002

Copyright: Elsevier

http://www.elsevier.com/

Postprint available at: Linköping University Electronic Press

http://urn.kb.se/resolve?urn=urn:nbn:se:liu:diva-133530

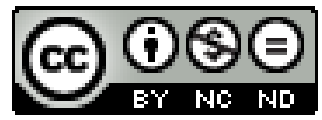




\section{Exploring the selectivity of $\mathrm{WO}_{3}$ with iridium catalyst in an ethanol/naphthalene mixture using multivariate statistics}

M. Bastuck ${ }^{a, b}$, D. Puglisi ${ }^{b}$, J. Huotaric, T. Sauerwalda ${ }^{a}$ J. Lappalainen ${ }^{c}$, A. Lloyd $^{2}$ Spetz $^{\text {b,c }}$, M. Andersson ${ }^{b, c}$, and A. Schütze ${ }^{a}$

${ }^{\mathrm{a}}$ Lab for Measurement Technology, Saarland University, D-66123, Saarbrücken, GERMANY.

bDiv. of Applied Sensor Science, Linköping University, SE-58183, Linköping, SWEDEN.

${ }^{\mathrm{c}}$ Faculty of Information Technology and Electrical Engineering, University of Oulu, FIN90014, Oulu, FINLAND.

Correspondence to: M. Bastuck (m.bastuck@lmt.uni-saarland.de, phone: +49 681302 4590, fax: +49681302 4665)

\section{Abstract}

Temperature cycled operation and multivariate statistics have been used to compare the selectivity of two gate (i.e. sensitive) materials for gas-sensitive, silicon carbide based field effect transistors towards naphthalene and ethanol in different mixtures of the two substances. Both gates have a silicon dioxide $\left(\mathrm{SiO}_{2}\right)$ insulation layer and a porous iridium (Ir) electrode. One of it has also a dense tungsten trioxide $\left(\mathrm{WO}_{3}\right)$ interlayer between Ir and $\mathrm{SiO}_{2}$. Both static and transient characteristics play an important role and can contribute to improve the sensitivity and selectivity of the gas sensor.

The $\mathrm{Ir} / \mathrm{SiO}_{2}$ is strongly influenced by changes in ethanol concentration, and is, thus, able to quantify ethanol in a range between 0 and 5 ppm with a precision of $500 \mathrm{ppb}$, independently of the naphthalene concentrations applied in this investigation. On the other hand, this 
sensitivity to ethanol reduces its selectivity towards naphthalene, whereas $\mathrm{Ir} / \mathrm{WO}_{3} / \mathrm{SiO}_{2}$ shows an almost binary response to ethanol. Hence, the latter has a better selectivity towards naphthalene and can quantify legally relevant concentrations down to $5 \mathrm{ppb}$ with a precision of $2.5 \mathrm{ppb}$, independently of a changing ethanol background between 0 and $5 \mathrm{ppm}$.

Keywords: metal oxide, pulsed laser deposition, silicon carbide field-effect transistor, SiCFET, gas sensor, temperature cycled operation, volatile organic compounds, quantification

\section{Introduction}

In the last two decades, gas-sensitive silicon carbide based field-effect transistors (SiC-FETs) have been extensively studied for applications in harsh environments, e.g. for exhaust monitoring [1-4]. Recently, their good sensitivity and selectivity towards volatile organic compounds (VOCs) in the low parts per billion (ppb) or even sub-ppb range $[5,6]$ has led to development towards the use in indoor air quality monitoring. Some of those VOCs have a major influence on the air quality as they are hazardous to human health already in very low concentrations. For example, naphthalene has a long-term exposure limit determined by the World Health Organization (WHO) of only $1.9 \mathrm{ppb}$ on annual average [7]. At the same time, there are other common VOCs which are not harmful up to very high concentrations (1000s of parts per million, ppm), like ethanol [8], but act as interfering gases in the intended sensor application. Indeed, ethanol can be commonly present in the low ppm-range indoors, where people on average spend more than $90 \%$ of their time [9]. This evidently shows that measuring Total VOC (TVOC), which is the prevalent measure for air quality nowadays, is not meaningful, and selective VOC detection and quantification are required.

To improve the selectivity of SiC-FETs, variations of the operation temperature, applied bias voltage as well as the sensing layers have shown to be effective approaches [10-13]. Since both the catalytic metal and the supporting insulator in the gate area of the device are active in 
the gas detection mechanism (more details are presented in section 2.1.2), both can be varied to increase the selectivity. It has been demonstrated that a stack of $\mathrm{SiO}_{2} / \mathrm{MgO} / \mathrm{LaF}_{3}$ or $\mathrm{IrO}_{2}$ improves the selectivity to oxygen [11], whereas a carbonate layer underneath platinum $\left(\mathrm{SiO}_{2} / \mathrm{MgO} / \mathrm{Pt} / \mathrm{Li}_{2} \mathrm{CO}_{3}-\mathrm{BaCO}_{3}\right)$ provides sensitivity to $\mathrm{CO}_{2}$ [12]. The hydrogen response of silicon based FET sensors employing different insulators $\left(\mathrm{SiO}_{2}, \mathrm{Si}_{3} \mathrm{~N}_{4}, \mathrm{Al}_{2} \mathrm{O}_{3} \mathrm{Ta}_{2} \mathrm{O}_{5}\right)$ and a platinum gate contact was related to the concentration of oxygen in the surface layer [13]. Here the traditional SiC-FETs with a sensitive layer of porous platinum (Pt) or iridium (Ir) as catalyst on dense silicon dioxide $\left(\mathrm{SiO}_{2}\right)$ insulator have been compared to a new generation of SiC-FETs with an additional layer of tungsten trioxide $\left(\mathrm{WO}_{3}\right)$ between Ir as catalyst and $\mathrm{SiO}_{2}$. The $\mathrm{Ir} / \mathrm{WO}_{3} / \mathrm{SiO}_{2} \mathrm{SiC}-\mathrm{FET}$ has been developed in order to improve the sensor's performance, i.e. sensitivity and selectivity towards VOCs. The catalytic properties of metal/metal-oxide compounds, and especially their good selectivity towards VOCs, have been regularly reported in literature [14-16].

In this work, two different kinds of SiC-FETs are exposed to gas mixtures with different ratios of ethanol and naphthalene: one with $\mathrm{WO}_{3}$ layer and porous $\mathrm{Ir}$ on top ("Ir/ $/ \mathrm{WO}_{3} / \mathrm{SiO}_{2}$ "), and one without $\mathrm{WO}_{3}$ layer (" $\mathrm{I} / \mathrm{SiO}_{2}$ ”). Driving them in temperature cycled operation gives the opportunity to explore the influence of temperature as well as using multivariate statistics for further analysis. All presented experiments and evaluations were performed related to a possible application of $\mathrm{Ir} / \mathrm{WO}_{3} / \mathrm{SiO}_{2}$ as selective sensing material for hazardous VOCs.

\section{Experimental setup}




\subsection{Transducer and operating modes}

\subsubsection{Temperature cycled operation}

Most materials used for sensing layers are not very specific to one compound. Instead, they will show similar reactions to many different substances, i.e. they have low selectivity. In the presence of several gases in several concentrations, and taking into account sensor drift, the static signal of one sensor does not carry enough information to allow discrimination or quantification of gases. Hence, this simple approach can only be employed in very controlled environments, but not in complex ones as, e.g., offices or homes. The selectivity can be improved by producing a signal pattern, either using a sensor-array of different sensors $[17,18]$, or the virtual-multisensor approach $[19,20]$, i.e. varying a parameter of the device to change its sensing properties, resulting in signals from "virtual different" sensors. In this work, the second approach has been used by varying the operation temperature of the sensor devices cyclically (temperature cycled operation, TCO). This operation mode has been shown to not only improve selectivity, but also sensitivity and stability for both MOS [19] and SiCFET [21] gas sensors.

\subsubsection{Silicon carbide based field-effect transistors}

The gate of the silicon carbide based field-effect transistor (SiC-FET, Figure 1a, produced by SenSiC AB, Kista, Sweden) is covered with the sensing material and eventually measures the shift in work function caused by dipoles on the metal/insulator interface. Those dipoles result from adsorption on the catalytic metal, decomposition and chemical reactions followed by spill-over of formed species to the insulator surface, exposed due to the porosity of the metal. The dipoles at the metal/insulator interface, which cause a shift in the work function may be hydrogen ions, i.e. protons, forming polar -OH groups with the gate oxide [22,23], changes in the surface coverage of negative oxygen ions by oxidizing or reducing gases $[4,24]$, or, 
arguably, adsorbed dipole molecules [25]. The coverage of the gate oxide with charges introduces an additional electrical field and, thus, affects the conducting channel between drain and source, which results in a change of resistance. This is determined as the current flowing when a voltage is applied over the drain-source contacts. Applying a relatively high $\mathrm{V}_{\mathrm{DS}}=4 \mathrm{~V}$ ensures that the device is in the saturation region, which has been shown to produce the largest signals [26]. The gate-source voltage, $\mathrm{V}_{\mathrm{GS}}$, is kept at zero at all times, which allows a current flow in the range of $\mu \mathrm{A}$ through the normally-on FET device.

The chip containing the transistor is glued to a Heraeus platinum heater, enabling electronic temperature control. As the thermal mass of the whole setup is relatively large with a $t_{90}$ of more than five seconds, transient effects in the sensing material are mostly masked by slow heating and cooling. Instead, the temperature cycle for this device focusses on different temperature plateaus, including temperatures that have been determined as optimal for several hazardous VOCs in preliminary experiments. The cycle includes plateaus of 200, 250 and $300{ }^{\circ} \mathrm{C}$ as well as a plateau at $100{ }^{\circ} \mathrm{C}$ to test the performance at low temperatures, and has a total length of $60 \mathrm{~s}$ (Figure 1b).

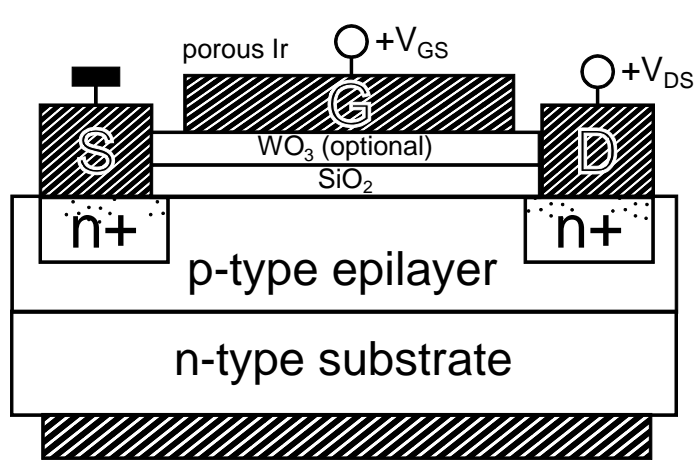

(a)

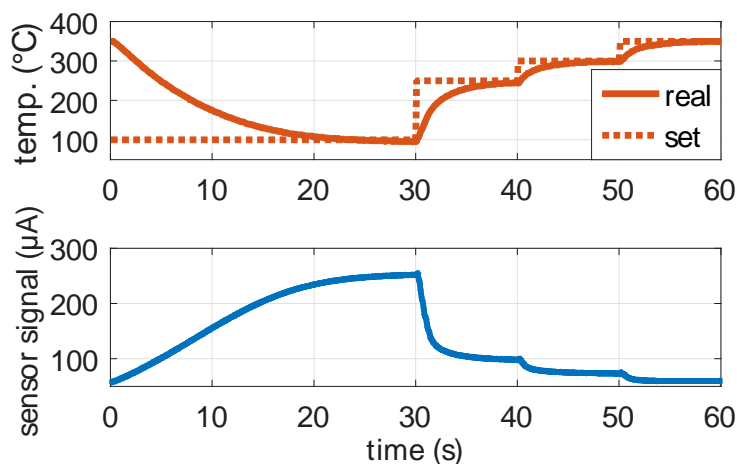

(b)

Figure 1: (a) Schematic cross-section of a SiC-FET device with an optional $\mathrm{WO}_{3}$ layer. (b) Temperature cycle and resulting sensor signal. 


\subsection{Preparation of sensitive layers}

A dense thin film of $\mathrm{WO}_{3}$ has been deposited on the gate area (onto the native $\mathrm{SiO}_{2}$ gate dielectrics) of the SiC-FET by pulsed laser deposition (PLD). The used laser was a XeClExcimer laser with operation at a wavelength of $308 \mathrm{~nm}$. The pulse length and repetition rate was $25 \mathrm{~ns}$ and $5 \mathrm{~Hz}$, respectively. The rotating target used was a pure ceramic $\mathrm{WO}_{3}$ disc. Before the deposition the vacuum chamber was pumped to a base pressure of $10^{-3} \mathrm{~Pa}$ and then an oxygen partial pressure of $5 \mathrm{~Pa}$ was injected to the deposition chamber. During deposition, the FET substrates were heated to $550{ }^{\circ} \mathrm{C}$ and the result was a dense, nanocrystalline, $\sim 50 \mathrm{~nm}$ thick layer of tungsten trioxide. Raman spectroscopy and grazing incidence X-ray diffraction (GIXRD) were used to determine the crystal structure of the $\mathrm{WO}_{3}$ layer. The layers were determined to be composed of monoclinic $\gamma$ - and $\varepsilon$-phases. The measured Raman spectrum of the layer is presented in Figure 2a. Contributions from both monoclinic $\gamma$ - and $\varepsilon$-phases can be identified from the curve. The peaks at wavenumbers 76, 93, 134, 272, 437, 639, 714, and $807 \mathrm{~cm}^{-1}$ are labelled in the literature as contributions from the $\mathrm{WO}_{3} \gamma$-phase [27], whereas the peaks at $142,272,304,425,643,677$, and $807 \mathrm{~cm}^{-1}$ are labelled as peaks characteristic to $\mathrm{WO}_{3} \varepsilon$-phase $[28,29]$. As it is seen, some of the peaks from the two phases are overlapping strongly making the labelling of the phonon modes challenging. However, there are also some peaks specific to the two different $\mathrm{WO}_{3}$ structures, thus enabling the phase identification of the $\mathrm{WO}_{3}$ structures from the spectrum. By using atomic force microscopy (AFM), the sample surfaces were also determined to be very flat. The average surface roughness value $R_{q}$ calculated from a $5 \mu \mathrm{m} \times 5 \mu \mathrm{m}$ AFM micrographs is $\mathrm{R}_{\mathrm{q}}=0.82 \mathrm{~nm}$. A $1 \mu \mathrm{m} \times 1 \mu \mathrm{m}$ AFM surface micrograph of the layer is shown in Figure 2b. It is seen that the flat surfaces of the layers are formed of very small nanosized grains. For the reference sample, the PLD processing step has been omitted. 


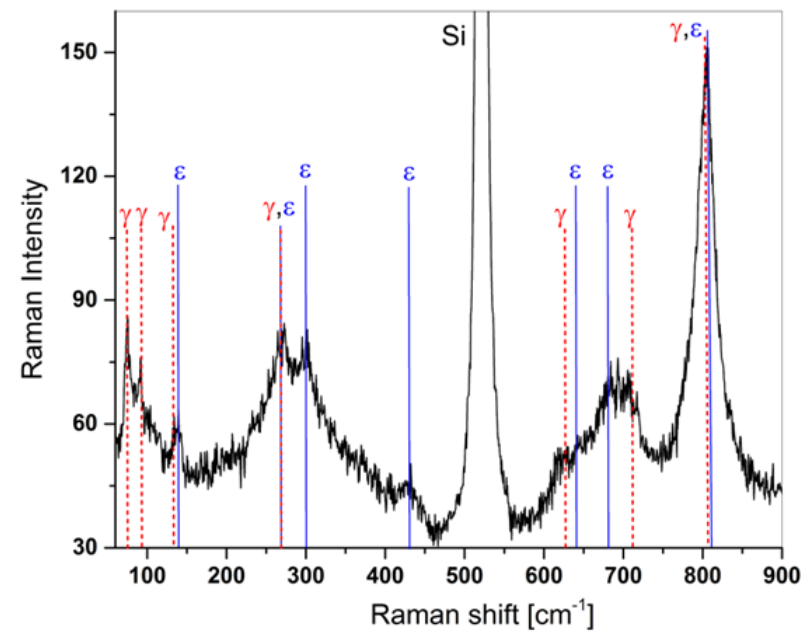

(a)

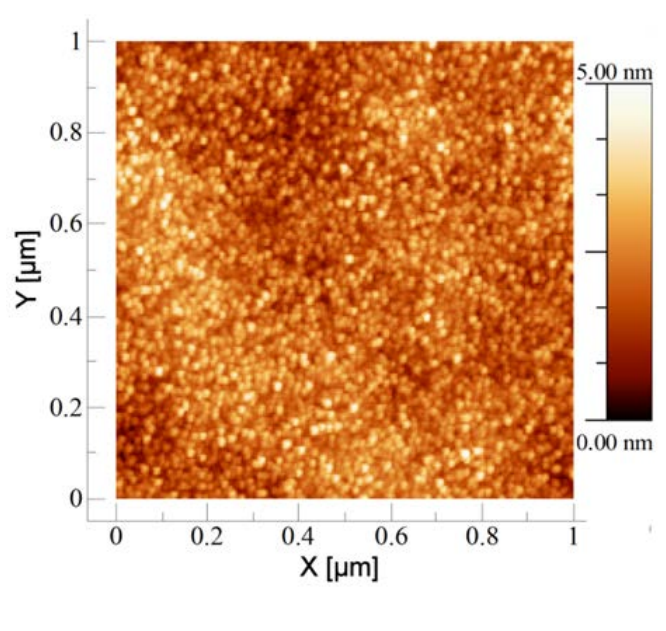

(b)

Figure 2: (a) Raman spectrum and (b) AFM $1 \mu \mathrm{m} \times 1 \mu \mathrm{m}$ surface micrograph of the PLD $\mathrm{WO}_{3}$ layer.

On top of the gate oxide $\left(\mathrm{WO}_{3}\right.$, or $\mathrm{SiO}_{2}$ for the reference sample), porous iridium with a thickness of $30 \mathrm{~nm}$ has been deposited by DC magnetron sputtering in Ar at a pressure of 50 mTorr (6.66 Pa).

\subsection{Measurement setup}

All experiments have been conducted with the measurement setup described in [30], which is able to reliably provide ppb-level concentrations of target gases reliably with very low contaminations. The sensors have been controlled and read-out using electronics developed and produced by 3S GmbH (Saarbrücken, Germany).

The SiC-FET was characterized using the gas exposure profile shown in Figure 3. It contains four repeated cycles of naphthalene concentrations from 5.0 to $40.0 \mathrm{ppb}$, in different ethanol backgrounds of 0.0, 1.0, 2.5 and 5.0 ppm, respectively. The carrier gas was humid air (80\% $\mathrm{N}_{2}, 20 \% \mathrm{O}_{2}, 50 \%$ relative humidity). Each gas exposure lasted for 30 minutes, or 30 temperature cycles presented in Figure 1b, of which the first five were not considered any further to account for the sensor’s response time. 

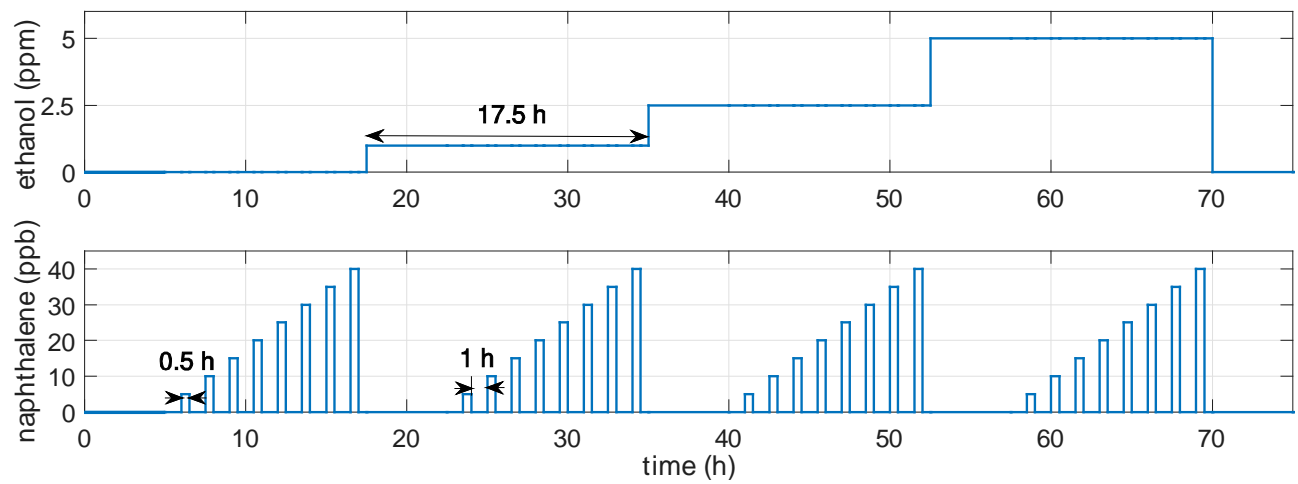

Figure 3: Gas exposure profile showing concentrations of ethanol (ppm) and naphthalene (ppb) over time. An exposure to naphthalene lasts 30 minutes and is followed by 60 minutes of carrier gas and current ethanol concentration without naphthalene.

\subsection{Data evaluation}

\subsubsection{Feature extraction and dimensionality reduction}

The SiC-FET sensor's signal was measured with $10 \mathrm{~Hz}$ sample rate, which results in 600 data points per temperature cycle. Most of those data points are highly correlated, violating one of the main assumptions, i.e. independency of the multivariate methods used for discrimination and quantification. This and other problems, summarized as "curse of dimensionality” [31], make reduction of each cycle's dimensionality, i.e. the number of data points, or "features", describing the signal, crucial. In a first step, this is done by manually selecting contiguous groups of data points ("ranges") and computing their mean and/or slope for all cycles. Depending on the number of ranges, this step will reduce the number of features by one to two orders of magnitude.

In this case, the means of 50 consecutive data points each were computed, resulting in 12 features. Additionally, each temperature plateau has been divided into 10 (for $100{ }^{\circ} \mathrm{C}$ ) or 5 (for all other temperatures) parts with logarithmically increasing length. Hence, one cycle is now being described by only 37 features instead of 600 features. 


\subsubsection{Discrimination}

Being able to discriminate one gas from all others in a complex mixture is a sign for good selectivity towards that gas. In this work, discrimination ability is tested using linear discriminant analysis (LDA, [32,33]) in combination with a $\mathrm{k}$ nearest neighbors (kNN) classifier. LDA is a method used in pattern recognition to find a linear combination of features that separates two or more classes. This is achieved by finding coefficients for all features so that the relation between inter-class variance and intra-class variance is maximized, where each "class" corresponds to the cycle running in a certain gas. This can also be understood as finding a lower-dimensional subspace in the multi-dimensional feature space which best discriminates the classes. This is another feature reduction step, and three or fewer dimensions, or “discriminant functions", are often enough to represent the data with negligible loss of information. The found coefficients are the outcome of "training" the algorithm with a specific dataset.

When an unknown cycle has to be classified, the value of each discriminant function is computed by taking the inner product of the feature vector and the vector of coefficients, which projects the cycle into the subspace. Subsequently, its k nearest neighbors are found in training data, and the majority class of those neighbors determines the class of the unknown data point. The parameter $\mathrm{k}$ is chosen based on the training data so that the best classification result is achieved.

\subsubsection{Quantification}

The basis for quantification is comprised of the same features used for discrimination. However, in this case, they are the input to partial least squares regression (PLSR, [34]), a powerful algorithm for regressing multivariate data. Similar to LDA, this algorithm also projects the features into a lower-dimensional subspace. However, it does so with the aim of 
finding the best compromise between minimizing the loss of explained variance and maximizing the correlation between projected and target (concentration) data. The training results in a set of coefficients and an offset; the predicted concentration for a set of features is the linear combination of the features with the determined coefficients and added offset.

Similarly to LDA, the number of components, i.e. the dimension of the subspace, can be chosen for PLSR. This choice is critical as wrong values can easily lead to under- or overfitted models. To determine the best number of components, the cross-validation root mean squared error (CV-RMSE) is computed for all possible numbers of components, and the model with the lowest CV-RMSE is chosen.

A proper identification of insignificant features, i.e. features that do not contribute to the model, has several advantages. Excluding them can make the model more stable as less noise is introduced. Also, the location of important features can highlight important parts of the cycle, and, thus, temperatures or temperature steps with high contribution to the quantification. Eventually, this can help to understand the processes happening on and in the material.

Testing features for significance is here done by applying the t-test [35] to the found coefficients. To do so, each coefficient is divided by its standard error, which, in turn, can be estimated by bootstrapping [36], i.e. sampling randomly from the data several times and building models from those samples. If the ratio lies below a distinct value, depending on chosen significance level and degrees of freedom in the model, the feature is called insignificant.

\subsubsection{Validation}

A model's prediction for its training data will become better for every feature that is added. However, this does not imply that its prediction ability for new data increases - in fact, it 
often gets worse since the model has adapted too much to small fluctuations and outliers in the training data. This state is called overfitting and has to be avoided to find a good model. One method to do this is to use k-fold cross-validation [37,38], in which each class is divided into k chunks. It will then train a model with (k-1) of these chunks and project the $\mathrm{k}^{\text {th }}$ chunk to simulate unknown data. For this unknown data, the error, i.e. either misclassification rate (in case of LDA) or RMSE (root mean squared error, in case of PLSR), is determined. This is done $\mathrm{k}$ times, until each chunk has been used as unknown data. The mean value of the $\mathrm{k}$ error values should be close to the error for the whole training data; a much higher cross-validation error indicates overfitting and an unsuitable model.

\section{Results}

\subsection{Discrimination}

In some cases, the exact quantity of a component is not of primary interest, but rather its presence at all. Such discrimination can determine whether a certain component is below or above a set threshold value. Ideally, a chemical sensor should have a strong response to the target component, i.e. high sensitivity, and low or no response to interfering components, i.e. high selectivity to the target component. For multivariate data, the discrimination model can be tuned to a specific target component by training it to discriminate all observations (here: temperature cycles) containing the target component (ethanol or naphthalene) against all observations that do not. The quality of the result of this discrimination will then give an idea about the sensor's sensitivity and selectivity for the specified target component, which are the better the less the model's response is influenced by variations of the interfering component.

Here we demonstrate discrimination of ethanol ("target component”) independently of its concentration or presence naphthalene (“interfering component”). An $\mathrm{Ir} / \mathrm{SiO}_{2}-\mathrm{FET}$ and an $\mathrm{Ir} / \mathrm{WO}_{3} / \mathrm{SiO}_{2}-\mathrm{FET}$ have been tested simultaneously. 
First, all cycles in $0 \mathrm{ppm}$ ethanol were pooled into one class which is to be discriminated against another class, made up of all cycles in more than 0 ppm ethanol, i.e. 1.0, 2.5 and $5.0 \mathrm{ppm}$. In both cases, this includes cycles in naphthalene concentrations between 0 and 40 ppb. The best projection to one dimension was found with LDA and validated with 10-fold cross-validation, as described in section 2.4. The results are shown in Figure 4.

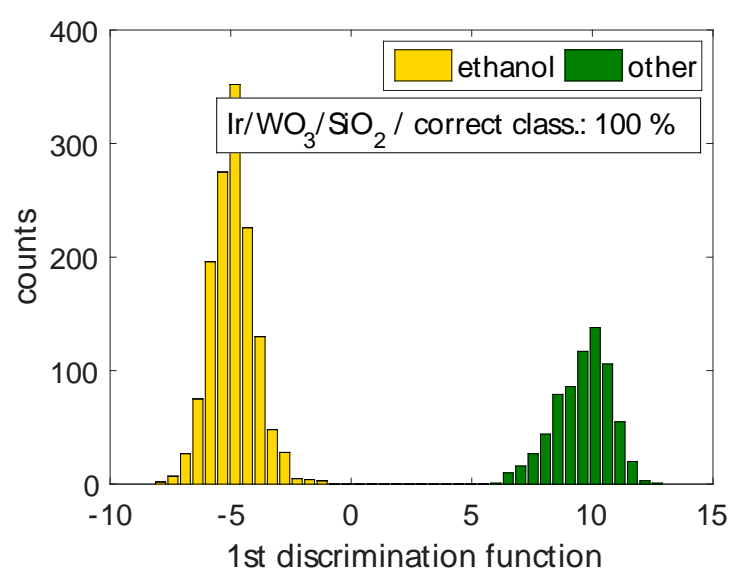

(a)

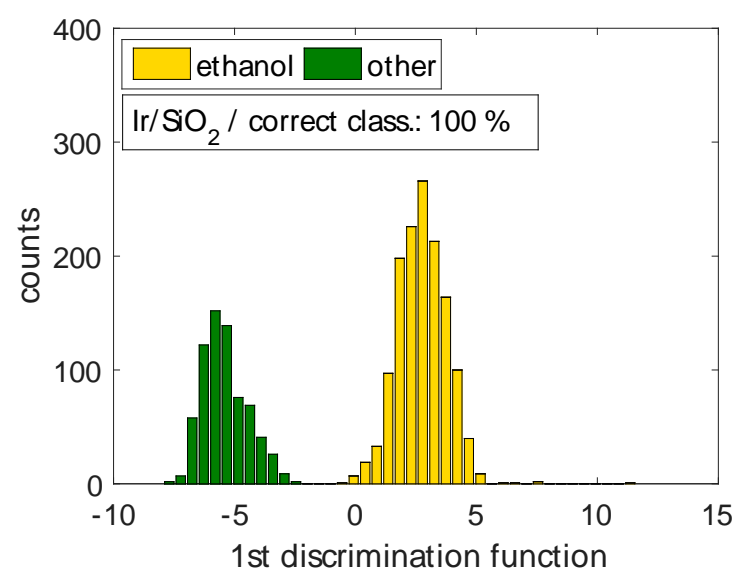

(b)

Figure 4: Discrimination of ethanol independent of presence or concentration of another component, i.e. naphthalene. The $\mathrm{Ir} / \mathrm{WO}_{3} / \mathrm{SiO}_{2}$-FET (a) shows a more pronounced separation of both classes compared to $\mathrm{Ir} / \mathrm{SiO}_{2}$-FET (b).

Both sensing layers, i.e. $\mathrm{Ir} / \mathrm{SiO}_{2}$ and $\mathrm{Ir} / \mathrm{WO}_{3} / \mathrm{SiO}_{2}$ are able to discriminate ethanol from nonethanol in all cases. However, $\mathrm{Ir} / \mathrm{WO}_{3} / \mathrm{SiO}_{2}$ produces a larger gap between both classes, which means a much clearer discrimination, especially for edge-cases. In Figure 5, another LDA has been performed with one class for each concentration, which reveals the "inner structure” of the previous diagrams. 

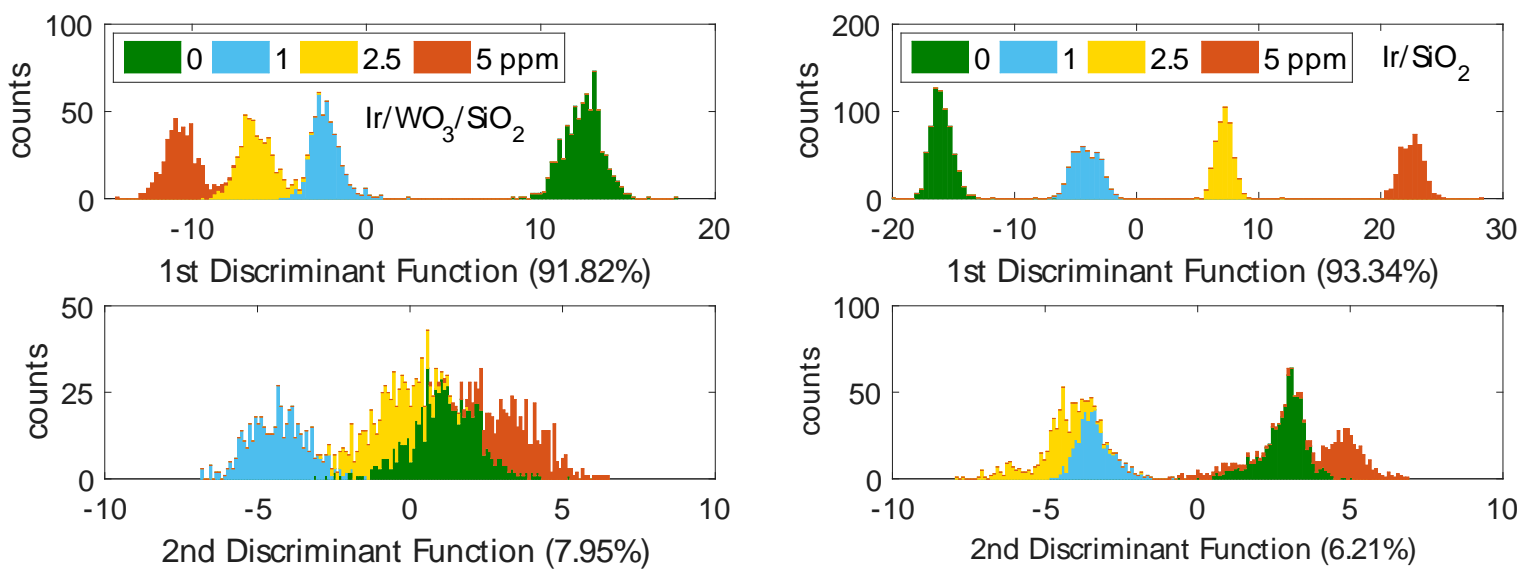

(a)

(b)

Figure 5: Discrimination of pure ethanol concentrations with (a) $\mathrm{Ir} / \mathrm{WO}_{3} / \mathrm{SiO}_{2}$ and (b) $\mathrm{Ir} / \mathrm{SiO}_{2}$. The first discriminant function, DF1, in (a) discriminates $0 \mathrm{ppm}$ clearly against all other concentrations, which are then discriminated further by the second discriminant function, DF2. In (b), DF1 discriminates all concentrations at once.

For $\mathrm{Ir} / \mathrm{WO}_{3} / \mathrm{SiO}_{2}$, the first discriminant function, which covers more than $90 \%$ of the overall signal information, separates especially $0 \mathrm{ppm}$ from every other concentration, while the distance between higher concentrations is only small in comparison. The second discriminant function then separates the remaining non-zero concentrations further. In contrast, for $\mathrm{Ir} / \mathrm{SiO}_{2}$, the majority of information is able to discriminate between all four concentrations at once. Hence, finding a projection in which all non-zero ethanol concentrations are located at the same point requires more of a compromise for $\mathrm{Ir} / \mathrm{SiO}_{2}$ than for $\mathrm{Ir} / \mathrm{WO}_{3} / \mathrm{SiO}_{2}$ which leads to the differences in class spacing in Figure 4.

For indoor air quality, detection of naphthalene independent of ethanol presence is more meaningful. Thus, in the following, the model was trained on the same data, but with naphthalene as target, and ethanol as interfering component. All cycles with presence of naphthalene were pooled into one class, and discriminated against cycles without 
naphthalene, independently of naphthalene or ethanol concentration. The results of this evaluation are shown in Figure 6.

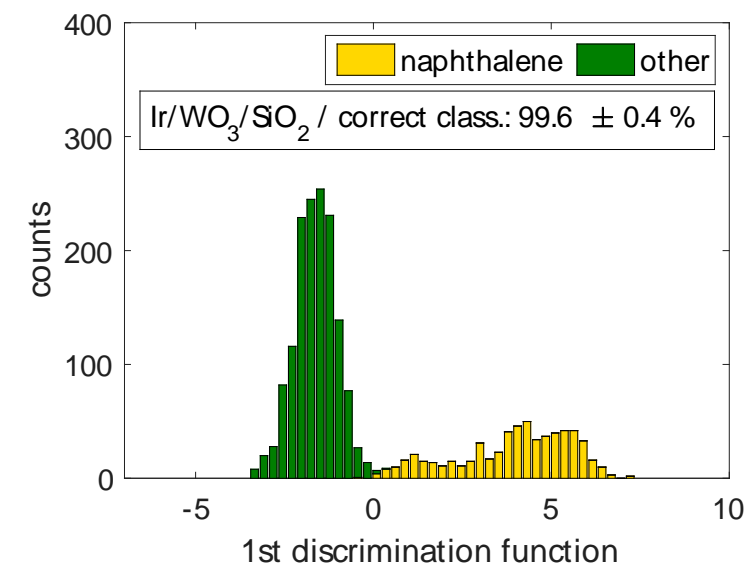

(a)

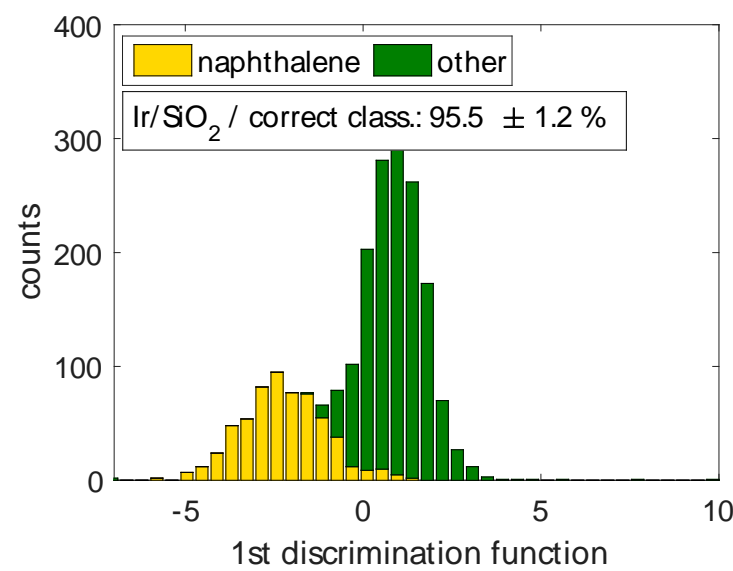

(b)

Figure 6: Discrimination of naphthalene independent of presence or concentration of another component, i.e. ethanol. Compared to $\mathrm{Ir} / \mathrm{WO}_{3} / \mathrm{SiO}_{2}$-FET (a), the $\mathrm{Ir} / \mathrm{SiO}_{2}$-FET (b) produces a large overlap of both classes, which is also expressed in the significantly worse classification rate.

It is evident that $\mathrm{Ir} / \mathrm{WO}_{3} / \mathrm{SiO}_{2}$ (Figure 6a) produces a significantly better separation of naphthalene from non-naphthalene than that in the case for $\mathrm{Ir} / \mathrm{SiO}_{2}$, which produces a large overlap of both classes. The best value for $\mathrm{k}$ of the kNN-classifier has in both cases been determined to be $\mathrm{k}=5$. With this $\mathrm{k}$-value, almost perfect classification is reached for $\mathrm{Ir} / \mathrm{WO}_{3} / \mathrm{SiO}_{2}$, while $\mathrm{Ir} / \mathrm{SiO}_{2}$ shows about $5 \%$ classification errors.

\subsection{Quantification}

When a more exact measure of the quantity of a certain component in a mixture is required, simple discrimination approaches cannot be used anymore. Those algorithms try to separate classes as far from each other as possible, which is less useful for concentration as a continuous measure, and, moreover, lacks interpolation ability. Instead, quantification algorithms like PLSR (described in section 2.4.3) can be employed. 
As before, selective quantification of ethanol is explored first, i.e. the algorithm's target variable is each cycle's ethanol concentration, ignoring naphthalene. The model is initially computed using all available 37 features. Then, the number of components with lowest CVRMSE is determined and the respective model is computed anew. Subsequently, the t-test is employed to discard non-significant features. In an iterative process, the new optimal number of components is determined; features are discarded, and so on. The process ends when no more features are discarded at a $5 \%$ significance threshold for a model with the optimal number of components. Those results and the respectively selected features (with a representative, not actual sensor response) are shown in Figure 7. The explained variance is given by the adjusted coefficient of determination, $\mathrm{R}^{2}$.
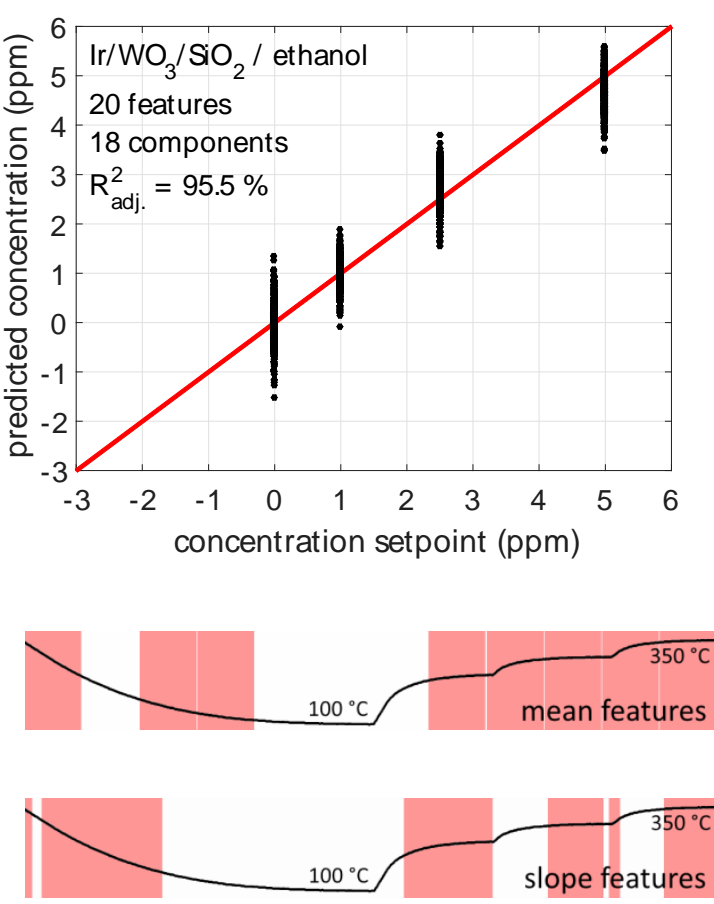

(a)
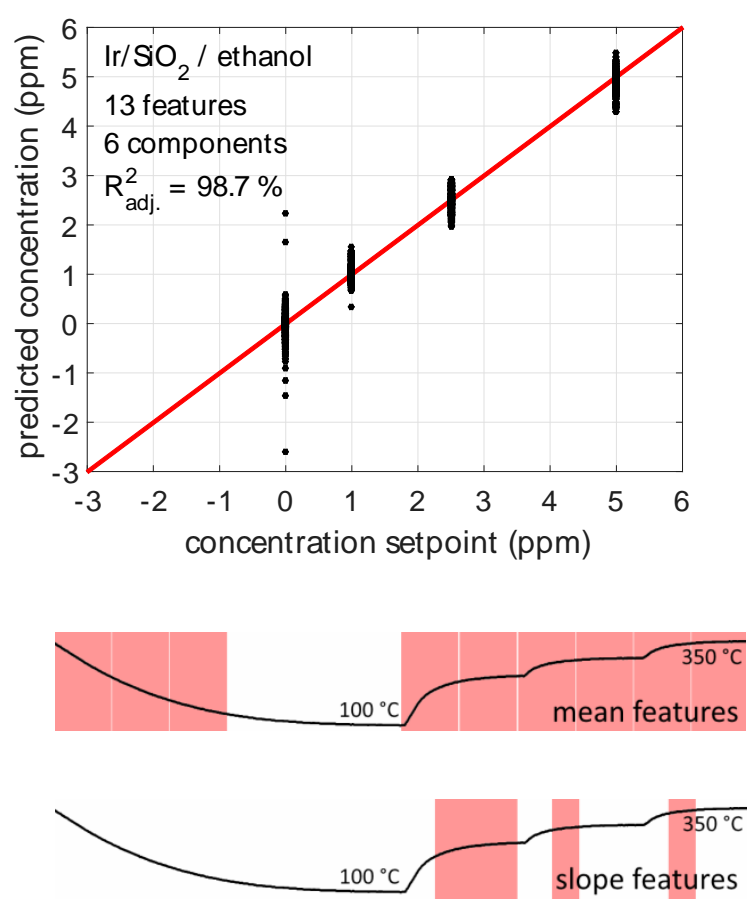

(b)

Figure 7: Quantification of ethanol independent of presence or concentration of another component, i.e. naphthalene. The precision for (a) $\mathrm{Ir} / \mathrm{WO}_{3} / \mathrm{SiO}_{2}$ is about $1 \mathrm{ppm}$, while for (b) $\mathrm{Ir} / \mathrm{SiO}_{2}$ it is about $0.5 \mathrm{ppm}$. The respectively chosen features and their corresponding sections of the cycle are highlighted. 
The precision of the model achieved for $\mathrm{Ir} / \mathrm{WO}_{3} / \mathrm{SiO}_{2}$, expressed as the approximate length of the bars of data points, is about $1 \mathrm{ppm}$, whereas it is $500 \mathrm{ppb}$ for $\mathrm{Ir} / \mathrm{SiO}_{2}$. Quantification of just ethanol without presence of naphthalene results in precisions of $500 \mathrm{ppb}$ and $150 \mathrm{ppb}$ for $\mathrm{Ir} / \mathrm{WO}_{3} / \mathrm{SiO}_{2}$ and $\mathrm{Ir} / \mathrm{SiO}_{2}$, respectively (data not shown). The superiority of $\mathrm{Ir} / \mathrm{SiO}_{2}$ is also expressed in the fact that it achieves a better $\mathrm{R}^{2}$ with fewer features and only 6 instead of 18 components, compared to $\mathrm{Ir} / \mathrm{WO}_{3} / \mathrm{SiO}_{2}$.

For both materials, all features are taken from temperatures above $100{ }^{\circ} \mathrm{C}$. The slopes of the transient signal during the transition from $100{ }^{\circ} \mathrm{C}$ to the first plateau at $200{ }^{\circ} \mathrm{C}$ are considered important by both models. Both, the "onset” of the flat part as well as the flat part itself seem to have predictive power. However, for the transitions to $300^{\circ} \mathrm{C}$ and $350{ }^{\circ} \mathrm{C}$, the slopes of the flat parts seem to provide more predictive power in the $\mathrm{Ir} / \mathrm{WO}_{3} / \mathrm{SiO}_{2}$ based PLSR model (Figure 7a), whereas the “onset” slopes contribute more in the $\mathrm{Ir} / \mathrm{SiO}_{2}$ model (Figure $7 \mathrm{~b}$ ).

Figure 8 shows the results for selective quantification of naphthalene in a similar way.
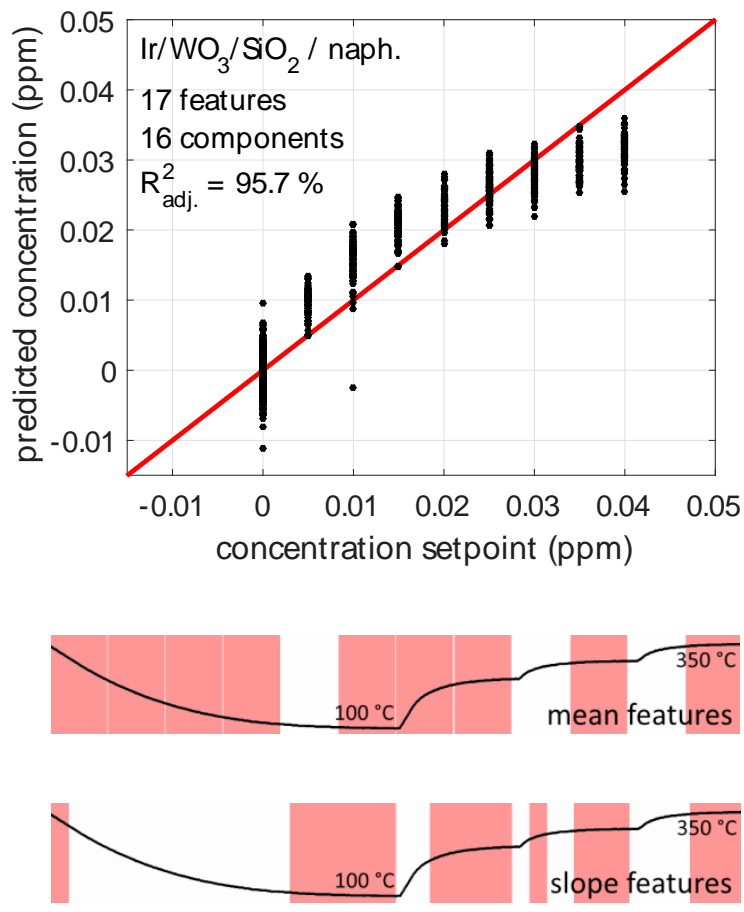

(a)
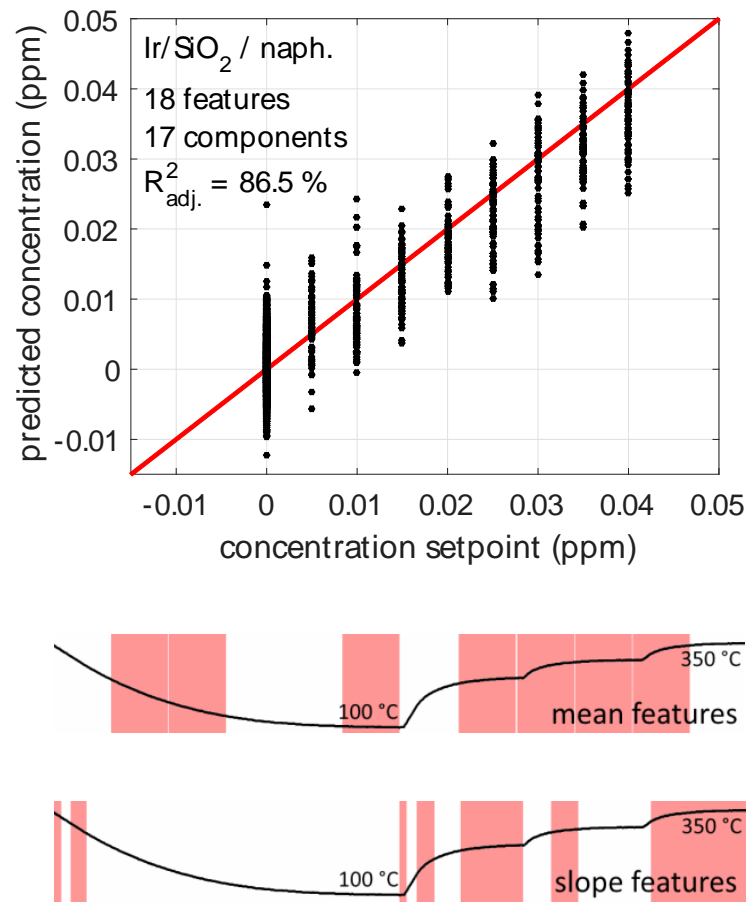

(b) 
Figure 8: Quantification of naphthalene independent of presence or concentration of another component, i.e. ethanol. $\mathrm{Ir} / \mathrm{WO}_{3} / \mathrm{SiO}_{2}$ (a) shows pronounced logarithmic dependence on the concentration, however, with better precision than the result for $\mathrm{Ir} / \mathrm{SiO}_{2}$ (b).

The signal of the $\mathrm{Ir} / \mathrm{WO}_{3} / \mathrm{SiO}_{2}$ device (a) shows a pronounced dependence on the logarithm of the concentration and thus very low accuracy (which cannot be captured well with $\mathrm{R}^{2}$ ), whereas the signal for $\mathrm{Ir} / \mathrm{SiO}_{2}$ (b) exhibits a linear dependence, but poorer precision (10 ppb) than $\mathrm{Ir} / \mathrm{WO}_{3} / \mathrm{SiO}_{2}(5 \mathrm{ppb})$.

In contrast to the models for ethanol, features at $100{ }^{\circ} \mathrm{C}$ are chosen in three of four cases, and slopes of the flat part of the plateaus get included in both models. The slope features from the transition from $100{ }^{\circ} \mathrm{C}$ to $250{ }^{\circ} \mathrm{C}$ are again included in both models, as before.

As the $\mathrm{Ir} / \mathrm{WO}_{3} / \mathrm{SiO}_{2}$ signal follows a logarithmic function, it can be linearized by training the model on logarithmic instead of actual concentration values. This has been done in Figure 9. A shift of 5 ppb has been applied to every concentration before taking the logarithm in order to avoid problems at $0 \mathrm{ppb}$. In the diagram, the real concentration values have been recalculated.

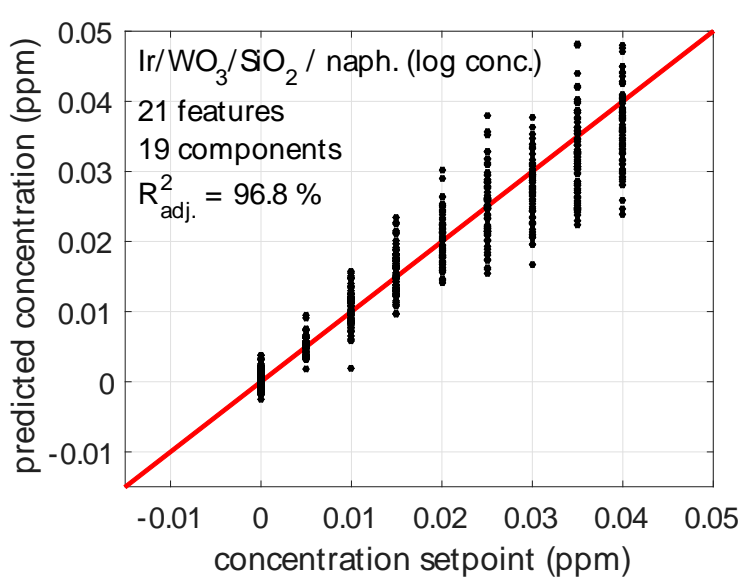

(a)
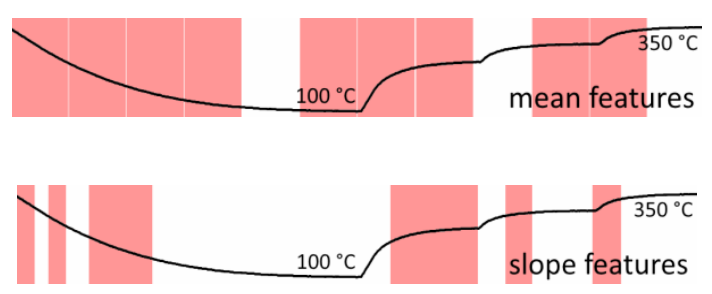

Figure 9: (a) The same PLSR model as in Figure 8a, but trained with the square root of concentrations to linearize the signal. (b) The features chosen by this model. 
The signal is now almost linear, therefore, interpolation between the discrete concentrations is assumed to be valid. Especially for low concentrations, the model exhibits the best precision reached in this work: about $2.5 \mathrm{ppb}$ at an absolute concentration of $5 \mathrm{ppb}$, in a varying ethanol background from 0 to $5 \mathrm{ppm}$. The precision decreases with absolute concentration to $7 \mathrm{ppb}$ at $40 \mathrm{ppb}$.

Also here, features at $100{ }^{\circ} \mathrm{C}$ and the signal slopes during the transition to $250{ }^{\circ} \mathrm{C}$ are chosen. For temperatures above $250{ }^{\circ} \mathrm{C}$, the picture of features for $\mathrm{Ir} / \mathrm{WO}_{3} / \mathrm{SiO}_{2}$ quantifying naphthalene Figure 9b looks similar to $\mathrm{Ir} / \mathrm{SiO}_{2}$ quantifying ethanol (Figure $7 \mathrm{~b}$ ), i.e., again the “onset” slopes show high predictive power.

\section{Discussion}

Both $\mathrm{Ir} / \mathrm{SiO}_{2}$ and $\mathrm{Ir} / \mathrm{WO}_{3} / \mathrm{SiO}_{2}$ can discriminate ethanol between 1 and $5 \mathrm{ppm}$ from no ethanol (Figure 4). This is an expected result since ethanol, especially in such relatively high concentrations, can usually be reliably detected by a broad range of gas sensor devices, including $\mathrm{Ir} / \mathrm{SiO}_{2}$-SiC-FETs. As ethanol concentration is 25 to 1000 times higher than naphthalene concentration, ethanol is likely to mask a portion of the sensor response to naphthalene, which makes the signal quite stable and independent from naphthalene. However, a comparison between Figure $6 \mathrm{a}$ and $\mathrm{b}$ reveals that addition of $\mathrm{a} \mathrm{WO}_{3}$ layer to the device noticeably widens the gap between the classes.

Indeed, an LDA of all ethanol concentration classes, as presented in Figure 5, shows that the step from 0 to $1 \mathrm{ppm}$ of ethanol causes a strong change for $\mathrm{Ir} / \mathrm{WO}_{3} / \mathrm{SiO}_{2}$, which is however followed by only small changes when the concentration is increased further. Hence, the material itself shows more of a binary response to ethanol. For $\mathrm{Ir} / \mathrm{SiO}_{2}$, instead, the signal change for each concentration step is similar, thus all concentrations can be separated from each other easily. This means, however, that a projection which can combine all these 
concentrations into one class cannot, at the same time, have a good separation to the noethanol class as well.

These findings can, in turn, explain the results for the classification problem "naphthalene vs. no naphthalene”, shown in Figure 6. For $\mathrm{Ir} / \mathrm{WO}_{3} / \mathrm{SiO}_{2}$ (Figure 6a), a small peak appears between 0 and 2 for the naphthalene class, which is followed by another, larger peak between 3 and 5. The small peak corresponds to naphthalene without ethanol background, while the larger peak corresponds to all naphthalene concentrations in any ethanol concentration from 1 to $5 \mathrm{ppm}$. On the other hand, $\mathrm{Ir} / \mathrm{SiO}_{2}$ (Figure 6b) is much more influenced by changing concentrations of ethanol, which leads to overlap of the classes. As the Ir layer deposited on top of the dense $\mathrm{WO}_{3}$ film is very porous, the new three-phase boundary between $\mathrm{WO}_{3} / \mathrm{Ir} /$ gas is believed to enhance the sensing properties of the FET structure towards VOCs, as compared to $\mathrm{SiO}_{2} / \mathrm{Ir} /$ gas three-phase boundary which is known to promote dissociation of e.g. ammonia [4]. It has already been shown by previous studies that PLD deposited $\mathrm{WO}_{3}$ layers can be used as extremely sensitive sensors for naphthalene [39].

An attempt was made to find a detection limit for naphthalene in varying ethanol concentrations for $\mathrm{Ir} / \mathrm{SiO}_{2}$. For this, the in each case lowest naphthalene concentration was shifted from the "naphthalene" to the "other" class (compare to Figure 6) until all concentrations up to $30 \mathrm{ppb}$ were contained in the "other" class. After each shift, the model was trained again; however, no significant improvement could be achieved, showing that the influence of changing ethanol concentration masks a large portion of the naphthalene influence.

In Figure 7, PLSR has been employed to perform quantification of the four ethanol concentrations (0, 1.0, 2.5 and $5.0 \mathrm{ppm})$. The results validate the previous findings. $\mathrm{Ir} / \mathrm{WO}_{3} / \mathrm{SiO}_{2}$ produces a model with a precision of about $1 \mathrm{ppm}$, compared to $500 \mathrm{ppb}$ for 
$\mathrm{Ir} / \mathrm{SiO}_{2}$. Obviously the binary response of $\mathrm{Ir} / \mathrm{WO}_{3} / \mathrm{SiO}_{2}$ to ethanol is a disadvantage for quantification. It should be noted that both models do not contain any features at the $100{ }^{\circ} \mathrm{C}$ plateau, showing that this temperature is too low to cause any significant reactions of ethanol on the sensor surface.

For naphthalene quantification (Figure 8) the $\mathrm{Ir} / \mathrm{WO}_{3} / \mathrm{SiO}_{2}$ benefits from its binary response to ethanol, which eventually lowers the ethanol's influence on the sensor signal. With $5 \mathrm{ppb}$, the achieved precision is about twice as good as for $\mathrm{Ir} / \mathrm{SiO}_{2}$. However, the $\mathrm{Ir} / \mathrm{WO}_{3} / \mathrm{SiO}_{2}$ also shows a distinct logarithmic dependence on the concentration. A response like this has been reported in literature and is explained by the Temkin isotherm [23]. The observation that all other models seem to exhibit a linear relationship can have two different explanations. In the case of ethanol quantification, the confined range of concentrations does not reveal a possible, non-linear dependence. For naphthalene quantification with $\mathrm{Ir} / \mathrm{SiO}_{2}$, the noise introduced by changing ethanol concentrations masks a large portion of the naphthalene reaction, and thus also its functional dependency.

In applications, the $\mathrm{Ir} / \mathrm{WO}_{3} / \mathrm{SiO}_{2}$ 's non-linear characteristic curve is undesired. However, it is easy to counteract by training the model with the logarithm of actual concentration values (Figure 9). The result is an almost linear response with especially good precision for low concentrations. At $5 \mathrm{ppb}$, the precision is about $2.5 \mathrm{ppb}$, independently of varying ethanol concentrations between 0 and 5 ppm. The corresponding precision in pure air, without ethanol variance, is as low as $1.5 \mathrm{ppb}$ (data not shown). Comparing this performance to other sensors is difficult as only very few studies on naphthalene detection are present in literature. However, we have recently shown that PLD deposited, porous $\mathrm{WO}_{3}$ performs similarly well on a MOS sensor platform [39]. In comparison to an optochemical naphthalene sensor 
described in [40], our sensor exhibits about 10 times longer response times (10 min vs. $1 \mathrm{~min}$ ), but an at least 100 times lower detection limit (5 ppb vs. 500 ppb).

All models for naphthalene quantification contain features from the $100{ }^{\circ} \mathrm{C}$ plateau, indicating that even such low temperatures can contribute to the model for naphthalene. At the same time, the sensor is not influenced by ethanol at such low temperatures, indicated by the fact that no features around $100{ }^{\circ} \mathrm{C}$ were chosen for ethanol quantification. Hence, this relatively low temperature contributes to discrimination of both gases and, thus, to the sensor's selectivity.

Low response to and, thus, low influence of ethanol is expected at low temperatures due to the reduced reaction rate of the molecule on the sensor surface. For pure $\mathrm{WO}_{3}$, increasing response to alcohols with increasing temperature has been reported in [41]. For naphthalene, on the other hand, features at $100{ }^{\circ} \mathrm{C}$ are contributing to the model, indicating significant influence of naphthalene also at low temperatures. One possible explanation could be that naphthalene adsorbs to the sensor surface and, while not reacting, blocks the adsorption site for other molecules. Hence, the influence of other gases like ethanol, oxygen and naturally occurring hydrogen on the sensor response is reduced, eventually changing the sensor signal on exposure to naphthalene. The raw signals hint to different sensing mechanisms for naphthalene for $\mathrm{Ir} / \mathrm{SiO}_{2}$ and $\mathrm{Ir} / \mathrm{WO}_{3} / \mathrm{SiO}_{2}$, respectively. This hypothesis must, however, be investigated in more detail.

All models include the slope of the flat part and its onset during the transition from $100{ }^{\circ} \mathrm{C}$ to $250{ }^{\circ} \mathrm{C}$. Moreover, the respective best models for ethanol (Figure $7 \mathrm{~b}, \mathrm{Ir} / \mathrm{SiO}_{2}$ ) and naphthalene (Figure 9a, $\mathrm{Ir} / \mathrm{WO}_{3} / \mathrm{SiO}_{2}$ ) prefer slope features from the "onset" region in the $300{ }^{\circ} \mathrm{C}$ and $350{ }^{\circ} \mathrm{C}$ plateaus. This result suggests that, despite slow heating and cooling of the device, the transient part of a temperature step still contains meaningful information. 


\section{Conclusions}

A comparative study of the performance of two SiC-FET gas sensor devices with different sensing layers has been presented. The selectivity of a flat and dense $\mathrm{WO}_{3}$ layer deposited as an additional oxide on top of $\mathrm{SiO}_{2}$ in the gate area of the transistor and covered with porous $\mathrm{Ir}$ ( $\left.\mathrm{Ir} / \mathrm{WO}_{3} / \mathrm{SiO}_{2}\right)$ towards the single components of an ethanol/naphthalene mixture has been explored and compared to the selectivity of pure Ir deposited directly on top of $\mathrm{SiO}_{2}$ $\left(\mathrm{Ir} / \mathrm{SiO}_{2}\right)$. Temperature cycled operation in combination with multivariate statistic methods like linear discriminant analysis and partial least squares regression have been used for the analysis and revealed that both static and dynamic signals contribute to selectivity.

$\mathrm{Ir} / \mathrm{SiO}_{2}$ reacts strongly to variations in ethanol concentration and is able to quantify concentrations between 0 and 5 ppm with a precision of $500 \mathrm{ppb}$, independently of a changing naphthalene concentration between 0 and $40 \mathrm{ppb}$. On the other hand, $\mathrm{Ir} / \mathrm{WO}_{3} / \mathrm{SiO}_{2}$ exhibits a more binary response to ethanol, making this material combination suitable for naphthalene detection and quantification in varying ethanol background. After linearization, a SiC-FET device coated with $\mathrm{Ir} / \mathrm{WO}_{3} / \mathrm{SiO}_{2}$ was able to quantify naphthalene concentrations up to $5 \mathrm{ppb}$ with a precision of $2.5 \mathrm{ppb}$, independently of a changing ethanol background between 0 and 5 ppm.

\section{Acknowledgement}

The authors would like to thank SenSiC AB, Sweden, for supplying the sensors. This project has received funding from the European Union's Seventh Framework Programme for research, technological development and demonstration under grant agreement No 604311.

\section{References}

[1] D.Y. Wang, L.M. Oberdier, Exhaust gas sensor diagnostic, US Patent 5,558,752, 1996. 
http://www.google.com/patents/US5558752.

[2] Z. Darmastuti, C. Bur, P. Möller, R. Rahlin, N. Lindqvist, M. Andersson, et al., SiCFET based SO2 sensor for power plant emission applications, Sensors Actuators, B Chem. 194 (2014) 511-520. doi:10.1016/j.snb.2013.11.089.

[3] M. Andersson, SiC based field effect sensors and sensor systems for combustion control applications, Linköping University, 2007.

[4] M. Andersson, R. Pearce, A.L. Spetz, New generation SiC based field effect transistor gas sensors, Sensors Actuators, B Chem. $179 \quad$ (2013) 95-106. doi:10.1016/j.snb.2012.12.059.

[5] C. Bur, M. Bastuck, D. Puglisi, A. Schütze, A. Lloyd Spetz, M. Andersson, Discrimination and quantification of volatile organic compounds in the ppb-range with gas sensitive SiC-FETs using multivariate statistics, Sensors Actuators B Chem. (2015) 1-9. doi:10.1016/j.snb.2015.03.016.

[6] D. Puglisi, J. Eriksson, C. Bur, A. Schütze, A. Lloyd Spetz, M. Andersson, Catalytic metal-gate field effect transistors based on SiC for indoor air quality control, J. Sensors Sens. Syst. 4 (2015) 1-8. doi:10.5194/jsss-4-1-2015.

[7] WHO Regional Office for Europe, WHO guidelines for indoor air quality, Geneva, 2010. doi:10.1186/2041-1480-2-S2-I1.

[8] J.M. Stellman, D. Osinsky, P. Markkanen, Ethanol, in: J.M. Stellman (Ed.), Encycl. Occup. Heal. Saf., 1998: p. 104.33.

[9] M.J. Jantunen, O. Hanninen, K. Katsouyanni, H. Knoppel, N. Kuenzli, E. Lebret, et al., Air pollution exposure in European cities: The "EXPOLIS" study, J. Expo. Anal. Environ. Epidemiol. 8 (1998) 495-518. 
http://www.scopus.com/inward/record.url?eid=2-s2.0-

2442770052\&partnerID=40\&md5=dc061e03f9e8e236d4e81d0f87a47c62.

[10] C. Bur, M. Bastuck, A.L. Spetz, M. Andersson, A. Schütze, Selectivity enhancement of SiC-FET gas sensors by combiningtemperature and gate bias cycled operation using multivariate statistics, Sensors Actuators, B Chem. 193 (2014) 931-940. doi:10.1016/j.snb.2013.12.030.

[11] M. Andersson, A. Lloyd Spetz, Tailoring of field effect gas sensors for sensing of nonhydrogen containing substances from mechanistic studies on model systems, in: 2009 IEEE Sensors, IEEE, 2009: pp. 2031-2035. doi:10.1109/ICSENS.2009.5398282.

[12] H. Inoue, M. Andersson, M. Yuasa, T. Kida, A. Lloyd Spetz, K. Shimanoe, CO2 sensor combining an MISiC capacitor and a binary carbonate, Electrochem. Solid-State Lett. 14 (2011) J4-J7. doi:10.1149/1.3512998.

[13] M. Eriksson, A. Salomonsson, I. Lundstr??m, D. Briand, A.E. ??bom, The influence of the insulator surface properties on the hydrogen response of field-effect gas sensors, J. Appl. Phys. 98 (2005) 1-7. doi:10.1063/1.1994941.

[14] M. Ferrandon, Mixed Metal Oxide - Noble Metal Catalysts for Total Oxidation of Volatile Organic Compounds and Carbon Monoxide, 2001.

[15] Y.S. Kim, Y.T. Kim, K. Lee, Low Temperature Volatile-Organic-Compound ( VOC ) Sensor Based on Tungsten Oxide Nanorods Preparation of Sensor Substrate Evaluation of Gas-Sensing Properties, Nanotech. 3 (2005) 169-172.

[16] Y. Li, X. Su, J. Jian, J. Wang, Ethanol sensing properties of tungsten oxide nanorods prepared by microwave hydrothermal method, Ceram. Int. 36 (2010) 1917-1920. doi:10.1016/j.ceramint.2010.03.016. 
[17] S.K. Jha, K. Hayashi, A novel odor filtering and sensing system combined with regression analysis for chemical vapor quantification, Sensors Actuators, B Chem. 200 (2014) 269-287. doi:10.1016/j.snb.2014.04.022.

[18] E.A. Baldwin, J. Bai, A. Plotto, S. Dea, Electronic noses and tongues: Applications for the food and pharmaceutical industries, Sensors. 11 (2011) 4744-4766. doi:10.3390/s110504744.

[19] M. Lösch, M. Baumbach, A. Schütze, Ozone detection in the ppb-range with improved stability and reduced cross sensitivity, Sensors Actuators, B Chem. 130 (2008) 367373. doi:10.1016/j.snb.2007.09.033.

[20] M. Leidinger, T. Sauerwald, W. Reimringer, G. Ventura, A. Schütze, Selective detection of hazardous VOCs for indoor air quality applications using a virtual gas sensor array, J. Sensors Sens. Syst. 3 (2014) 253-263. doi:10.5194/jsss-3-253-2014.

[21] C. Bur, M. Andersson, A.L. Spetz, N. Helwig, A. Schütze, Detecting Volatile Organic Compounds in the ppb range with platinum-gate SiC-Field Effect Transistors, in: IEEE SENSORS $2013 \quad-\quad$ Proc., IEEE, 2014: pp. 3221-3228. doi:10.1109/ICSENS.2013.6688279.

[22] I. Lundström, M.S. Shivaraman, C.M. Svensson, A hydrogen-sensitive Pd-gate MOS transistor, J. Appl. Phys. 46 (1975) 3876-3881. doi:10.1063/1.322185.

[23] M. Eriksson, L. Ekedahl, Hydrogen adsorption states at the Pd/SiO2 interface and simulation of the response of a Pd metal-oxide-semiconductor hydrogen sensor, J. Appl. Phys. 83 (1998) 3947-3951. doi:10.1063/1.367150.

[24] J. Schalwig, P. Kreisl, S. Ahlers, G. Müller, Response mechanism of SiC-based MOS field-effect gas sensors, IEEE Sens. J. 2 (2002) 394-402. 
doi:10.1109/JSEN.2002.806214.

[25] I. Lundström, H. Sundgren, F. Winquist, M. Eriksson, C. Krantz-Rülcker, A.L. Spetz, Twenty-five years of field effect gas sensor research in Linköping, Sensors Actuators, B Chem. 121 (2007) 247-262. doi:10.1016/j.snb.2006.09.046.

[26] D. Puglisi, J. Eriksson, M. Andersson, J. Huotari, M. Bastuck, C. Bur, et al., Exploring the Gas Sensing Performance of Catalytic Metal/Metal Oxide 4H-SiC Field Effect Transistors (accepted), Mater. Sci. Forum. (2016).

[27] E. Cazzanelli, C. Vinegoni, G. Mariotto, A. Kuzmin, J. Purans, Low-Temperature Polymorphism in Tungsten Trioxide Powders and Its Dependence on Mechanical Treatments, J. Solid State Chem. 143 (1999) 24-32. doi:10.1006/jssc.1998.8061.

[28] A. Baserga, V. Russo, F. Di Fonzo, A. Bailini, D. Cattaneo, C.S. Casari, et al., Nanostructured tungsten oxide with controlled properties: Synthesis and Raman characterization, Thin Solid Films. $515 \quad$ (2007) 6465-6469. doi:10.1016/j.tsf.2006.11.067.

[29] L. Wang, A. Teleki, S.E. Pratsinis, P.I. Gouma, Ferroelectric WO 3 Nanoparticles for Acetone Selective Detection, Chem. Mater. $20 \quad$ (2008) 4794-4796. doi:10.1021/cm800761e.

[30] N. Helwig, M. Schüler, C. Bur, A. Schütze, T. Sauerwald, Gas mixing apparatus for automated gas sensor characterization, Meas. Sci. Technol. 25 (2014) 055903. doi:10.1088/0957-0233/25/5/055903.

[31] C. Böhm, S. Berchtold, D. Keim, Searching in high-dimensional spaces: Index structures for improving the performance of multimedia databases, ACM Comput. Surv. 33 (2001) 322-373. doi:10.1145/502807.502809. 
[32] E.N. Kuntsche, E. Backhaus, B. Erichson, W. Plinke, R. Weiber, B.G. Tabachnick, et al., Multivariate Analysemethoden: Eine anwendungsorientierteEinführung., 9th ed., Springer, Berlin, 2001. doi:10.1024//1421-0185.60.4.279.

[33] R. Gutierrez-Osuna, Pattern analysis for machine olfaction: A review, IEEE Sens. J. 2 (2002) 189-202. doi:10.1109/JSEN.2002.800688.

[34] S. Wold, M. Sjöström, L. Eriksson, PLS-regression: A basic tool of chemometrics, Chemom. Intell. Lab. Syst. 58 (2001) 109-130. doi:10.1016/S0169-7439(01)00155-1.

[35] Student, Probable Error of a Mean, Biometrika. 6 (1908) 1-25. doi:10.2307/2331554.

[36] B. Efron, Bootstrap Methods: Another Look at the Jackknife, Ann. Stat. 7 (1979) 1-26. doi:10.1214/aos/1176344552.

[37] M. Browne, Cross-Validation Methods., J. Math. Psychol. 44 (2000) 108-132. doi:10.1006/jmps.1999.1279.

[38] D. Osten, Selection of optimal regression models via cross-validation, J. Chemom. 2 (1988) 39 - 48. doi:10.1002/cem.1180020106.

[39] M. Leidinger, J. Huotari, T. Sauerwald, J. Lappalainen, A. Schütze, Selective detection of naphthalene with nanostructured WO3 gas sensors prepared by pulsed laser deposition, J. Sensors Sens. Syst. 5 (2016) 147-156. doi:10.5194/jsss-5-147-2016.

[40] M. Girschikofsky, M. Rosenberger, S. Belle, M. Brutschy, S.R. Waldvogel, R. Hellmann, Highly Sensitive Detection of Naphthalene in Solvent Vapor Using a Functionalized PBG Refractive Index Sensor, Sensors. 12 (2012) 2018-2025. doi:10.3390/s120202018.

[41] D. Kohl, L. Heinert, J. Bock, T. Hofmann, P. Schieberle, Systematic studies on responses of metal-oxide sensor surfaces to straight chain alkanes, alcohols, aldehydes, 
ketones, acids and esters using the SOMMSA approach, Sensors Actuators B Chem. 70 (2000) 43-50. doi:10.1016/S0925-4005(00)00552-9. 